\title{
Weakened Center-Surround Interactions in Visual Motion Processing in Schizophrenia
}

\author{
Duje Tadin, Jejoong Kim, Mikisha L. Doop, Crystal Gibson, Joseph S. Lappin, Randolph Blake, and Sohee Park \\ Vanderbilt Vision Research Center and Department of Psychology, Vanderbilt University, Nashville, Tennessee 37203
}

\begin{abstract}
Schizophrenia is often accompanied by a range of visual perception deficits, with many involving impairments in motion perception. The presence of perceptual abnormalities may impair neural processes that depend on normal visual analysis, which in turn may affect overall functioning in dynamic visual environments. Here, we examine the integrity of suppressive center-surround mechanisms in motion perception of schizophrenic patients. Center-surround suppression has been implicated in a range of visual functions, including figureground segregation and pursuit eye movements, visual functions that are impaired in schizophrenia. In control subjects, evidence of center-surround suppression is found in a reduced ability to perceive motion of a high-contrast stimulus as its size increases. This counterintuitive finding is likely a perceptual correlate of center-surround mechanisms in cortical area MT. We now show that schizophrenic patients exhibit abnormally weak center-surround suppression in motion, an abnormality that is most pronounced in patients with severe negative symptoms. Interestingly, patients with the weakest surround suppression outperformed control subjects in motion discriminations of large high-contrast stimuli. This enhanced motion perception of large high-contrast stimuli is consistent with an MT abnormality in schizophrenia and has a potential to disrupt smooth pursuit eye movements and other visual functions that depend on unimpaired center-surround interactions in motion.
\end{abstract}

Key words: schizophrenia; perception deficit; visual motion; center-surround; inhibition; negative symptoms

\section{Introduction}

Schizophrenia is associated with a number of abnormalities in visual perception, including deficits of visual backward masking (Green and Walker, 1986; Schechter et al., 2003), luminance flicker sensitivity (Slaghuis and Bishop, 2001), biological motion perception (Kim et al., 2005), and velocity discrimination (Chen et al., 1999a). Visual deficits in schizophrenia often share a common thread: abnormalities are found in perceiving moving and/or dynamic stimuli, stimulus conditions implicating impairments of the magnocellular (transient) visual processing stream (Butler and Javitt, 2005). This broad class of deficits may cascade into impaired functioning in dynamic visual environments. Moreover, other brain functions that rely on transient information processed by the visual system may also be jeopardized. One notable example is the smooth pursuit eye movement (SPEM) deficit, present in a majority of schizophrenic patients. This deficit is expressed as an abnormally low pursuit gain and frequent saccadic intrusions (Holzman et al., 1973). The magnitude of SPEM deficit is correlated with the motion perception abnormal-

\footnotetext{
Received June 20, 2006; revised Aug. 22, 2006; accepted Sept. 27, 2006.

This work was supported by National Institutes of Health Grants MH58406 and MH073028 (S.P.), EY07760 (R.B.), and EY015558 (J.S.L.), National Alliance for Research on Schizophrenia and Depression Independent Investigator Award (S.P.), and Vision Core Grant EY08126. We thank Caroline Davis for assistance in recruiting and testing subjects.

Correspondence should be addressed to either Duje Tadin or Sohee Park, 301 Wilson Hall, Department of Psychology, Vanderbilt University, Nashville, TN 37203. E-mail: duje.tadin@vanderbilt.edu or sohee.park@vanderbilt.edu.

DOI:10.1523/JNEUROSCI.2592-06.2006

Copyright $\odot 2006$ Society for Neuroscience $\quad$ 0270-6474/06/2611403-10\$15.00/0
}

ities (Chen et al., 1999b,c; Slaghuis et al., 2005), suggesting a possible link between two deficits.

Intimately involved in cortical motion processing are visual areas MT and MST (Orban, 1997; Born and Bradley, 2005), key brain areas in the magnocellular stream. It is natural to speculate, therefore, that areas MT/MST may be abnormal in schizophrenia (Chen et al., 2003a). However, it is yet unclear what mechanisms underlie this deficit. One functional role of MT is representation of both object and background motion (Born and Bradley, 2005). This dual coding of motion can be linked to two types of MT neurons: antagonistic center-surround neurons, which have inhibitory surrounds and respond best to small moving objects, and wide-field neurons, which lack inhibitory surrounds and prefer large moving fields (Allman et al., 1985; Born and Tootell, 1992). Furthermore, microstimulation of MT sites with centersurround neurons shifts SPEM in the direction similar to the preferred direction of the stimulated clusters of neurons (Born et al., 2000), suggesting that center-surround neurons encode object motion. In contrast, microstimulation of MT sites with wide-field neurons shifts SPEM in the direction opposite to the preferred direction of the stimulated neurons, suggesting that wide-field neurons encode background motion. Thus, any abnormality of center-surround interactions has a potential to disrupt normal SPEM.

In human vision, antagonistic center-surround interactions are revealed by the reduced ability to perceive motion as the size of a moving stimulus increases (Tadin et al., 2003; Paffen et al., 2006). This observation suggests the presence of suppressive surround mechanisms. Moreover, both behavioral (Tadin et al., 2003) and neurophysiological (Pack et al., 2005) investigations 
Table 1. The demographic data

\begin{tabular}{lcc}
\hline & $\begin{array}{c}\text { Control subjects } \\
(n=14)\end{array}$ & $\begin{array}{l}\text { Schizophrenic subjects } \\
(n=16)\end{array}$ \\
\hline Age & $35.5(7.2)$ & $35.9(8.8)$ \\
Education (years) & $14.3(1.9)$ & $12.9(1.9)$ \\
Estimated full-scale IQ $^{a, b}$ & $100.3(15.9)$ & $100.4(16.1)$ \\
BPRS & $\mathrm{N} / \mathrm{A}$ & $24.8(16.1)$ \\
SANS & $\mathrm{N} / \mathrm{A}$ & $28.6(18.8)$ \\
SAPS & $\mathrm{N} / \mathrm{A}$ & $25.1(23.5)$ \\
SPQ & $10.7(6.2)$ & $\mathrm{N} / \mathrm{A}$ \\
Global handedness score & $70.4(56.1)$ & $59.1(43.9)$ \\
CPZ equivalent $^{c}$ & $\mathrm{~N} / \mathrm{A}$ & $325.0(192.0)$ \\
IInness duration & $\mathrm{N} / \mathrm{A}$ & $13.4(7.1)$ \\
Zigler score $^{e}$ & $6.0(1.5)$ & $2.6(1.1)$ \\
\hline
\end{tabular}

\section{Mean (SD) are shown. N/A, Not applicable.}

${ }^{a}$ Wechsler Adult Intelligence Scale, Revised (Wechsler, 1981).

${ }^{b}$ For subject JY, the Barona Index (Spreen and Strauss, 1998) was used to compute estimated full-scale IQ because English was not this subject's native language.

'Global Handedness Questionnaire (Ransil and Schachter, 1994).

${ }^{d}$ Chlorpromazine dose equivalent (in milligrams).

'Zigler Score of Social Functioning (Zigler and Levine, 1981).

have shown that surround suppression only occurs with highcontrast stimuli. Thus, investigating perceptual center-surround interactions in motion perception should elucidate the integrity of neural center-surround interactions (Tadin and Lappin, 2005) and contribute to our understanding of motion perception deficit(s) in schizophrenia. In the context of schizophrenia, abnormal center-surround interactions would suggest the involvement of cortical area MT in those abnormalities.

\section{Materials and Methods}

\section{Motion discrimination experiment}

Subjects. Sixteen patients (four females) who met the Diagnostic and Statistical Manual of Mental Disorders IV (DSM-IV) criteria for schizophrenia were recruited from an outpatient treatment facility in Nashville, TN. Their diagnosis was determined from the Structured Clinical Interview for DSM-IV (Spitzer and Williams, 1985). The mean age of the patients was 35.9 years (SD, 8.8 years), mean education level was 12.9 years (SD, 1.9 years), and they had been ill for an average of 13.4 years (SD, 7.1 years). All patients were on atypical antipsychotic medication at the time of the experiment. Mean chlorpromazine equivalent (CPZ) dose was $325 \mathrm{mg} / \mathrm{d}$ (SD, 192). Clinical symptoms were assessed with the Brief Psychiatric Rating Scale (BPRS) (Overall and Gorham, 1962), the Scale for Assessment of Positive Symptom (SAPS) (Andreasen and Olsen, 1982), and the Scale for Assessment of Negative Symptom (SANS) (Andreasen and Olsen, 1982). Mean scores for BPRS, SAPS, and SANS were 24.8 (SD, 16.1), 25.1 (SD, 23.5), and 28.6 (SD, 18.8), respectively. Median scores for BPRS, SAPS, and SANS were 22.5, 18, and 28.5, respectively (Table 1).

Fourteen healthy control subjects (six females) with no history of mental illness or neurological disorders were recruited from the community. Their mean age was 35.5 years (SD, 7.2 years), and their mean education level was 14.3 years (SD, 1.9 years). Normal control subjects were screened to rule out schizotypal personality using the Schizotypal Personality Questionnaire (SPQ) (Raine, 1991) before the experiment. No control subject scored high on the SPQ; the mean score on the SPQ was 10.7 (SD, 6.2) of 72. No control subject was receiving psychotropic medications. There was no significant difference between groups in age $\left(t_{(26)}=0.12 ; p=0.90\right)$.

The experimental protocol was approved by the Institutional Review Board of Vanderbilt University. All subjects were given adequate information to insure that they understood the consent procedure before they were recruited. All subjects were paid for their participation.

Psychophysical task. Stimulus patterns were created in MatLab with the Psychophysics Toolbox (Brainard, 1997) and Video Toolbox (Pelli, $1997)$ and shown on a linearized video monitor $(800 \times 600$ pixels reso-

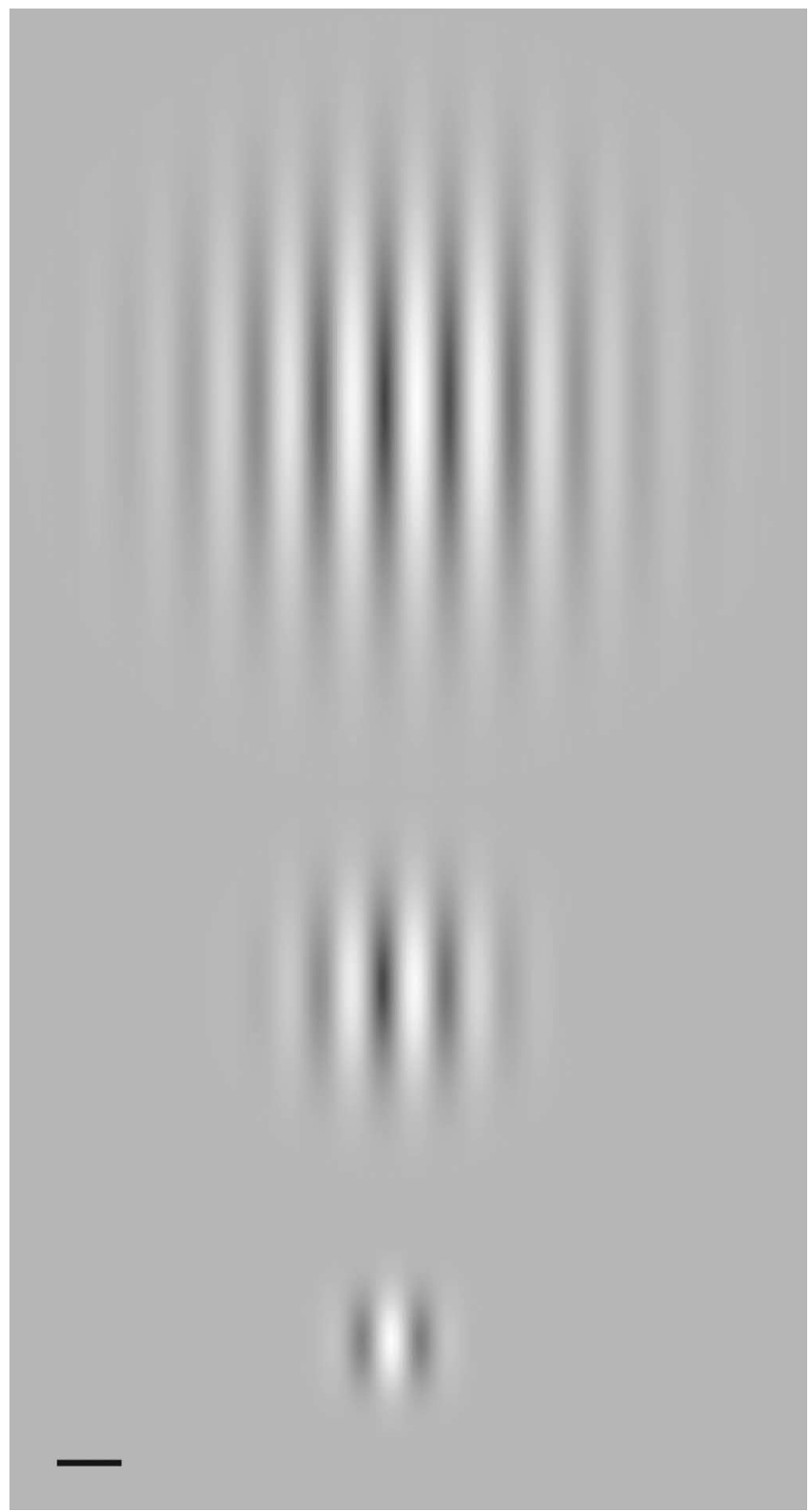

Figure 1. Examples of three different stimulus sizes used in the motion discrimination task. Scale bar, $1^{\circ}$. These Gabor patches consisted of a stationary Gaussian spatial window and dark/ white bars that drifted either rightward or leftward.

lution; $120 \mathrm{~Hz}$ ). Viewing was binocular at $83 \mathrm{~cm}$. The ambient illumination was $4.8 \mathrm{~cd} / \mathrm{m}^{2}$, and the background luminance was $60.5 \mathrm{~cd} / \mathrm{m}^{2}$.

The motion stimulus was a standard Gabor patch stimulus, a drifting vertical sine grating windowed by a stationary two-dimensional Gaussian envelope (Fig. 1). The size was defined as 2 SDs of the spatial Gaussian window. Stimulus duration was controlled by a temporal Gaussian envelope. This allowed brief presentations of moving stimuli. Duration was defined as 2 SDs of the temporal Gaussian. Subjects were instructed to foveate the fixation cross and initiate each trial by a key press. Then, after $600 \mathrm{~ms}$, a drifting Gabor patch was presented foveally and subjects indicated the perceived direction (left vs right) by a key press. Positive feedback was provided. Spatial frequency was 1 cycle $/{ }^{\circ}$ and speed was $4^{\circ} / \mathrm{s}(4$ $\mathrm{Hz})$. Three stimulus sizes $\left(2 \sigma=1,2\right.$, and $\left.4^{\circ}\right)$ at two contrasts $(2.8$ and $42 \%$ ) were investigated, yielding six conditions (Fig. 1). In the text, these three stimulus sizes are also referred to as small, medium, and large. The stimulus sizes were selected so that the average receptive field size in foveal MT falls somewhere between small and medium stimuli. Foveal receptive size estimates for macaque MT range from radius of $0.6^{\circ}(\mathrm{Al}-$ 
bright and Desimone, 1987) to radius of $2.6^{\circ}$ (Raiguel et al., 1995). As a comparison, $\mathrm{V} 1$ receptive fields tend to be $\sim 10$ times smaller than MT receptive fields at a similar eccentricity (Gattass and Gross, 1981; Albright and Desimone, 1987). Thus, our medium stimuli $\left(2 \sigma=2^{\circ}\right)$ are likely large enough to stimulate some surrounds in the foveal region of MT, and are more than an order of magnitude larger than V1 receptive fields. Moreover, our large moving stimuli $\left(2 \sigma=4^{\circ}\right)$ should stimulate surrounds of most foveal center-surround neurons in MT. Of course, this statement assumes that the properties of human and macaque MT are comparable (Rees et al., 2000) and that the receptive field sizes are similar for two species (Kastner et al., 2001).

Duration thresholds ( $82 \%$ ) for perceiving stimulus motion (i.e., minimum stimulus duration required for $82 \%$ correct performance) were estimated in each block of trials by two interleaved QUEST staircases (Watson and Pelli, 1983). Based on a subject's responses, the QUEST staircase method adaptively adjusts $\log _{10}$ of the stimulus duration. Accordingly, all analyses were performed on $\log _{10}$ duration thresholds.

Use of duration thresholds was based on the assumption that if the neural response to a stimulus is weak and/or noisy, then longer stimulus exposure will be required for correct perception. More specifically, deciding whether an object is moving in one of two possible directions can be conceptualized as a process involving accumulation of sensory evidence over time (Gold and Shadlen, 2000; Roitman and Shadlen, 2002). When neuronal responses are noisy or attenuated, as with a highly suppressed motion stimulus, sensory evidence accumulates more slowly and a correct decision thus will require longer exposure duration (Roitman and Shadlen, 2002).

Use of the staircase procedure ensured that both control subjects and schizophrenic patients performed at the same level of accuracy. Adjustment of task difficulty with an adaptive staircase such as the QUEST (especially with a conservative $82 \%$ threshold) minimizes the experience of failure, which presumably should have a positive effect on subjects' motivation. For each condition, four blocks of trials were completed, with results of the first block discarded as practice. The order of conditions was randomized. The completion of the study required two $1 \mathrm{~h}$ sessions.

We were particularly interested in whether subjects' performance improves or deteriorates with increasing stimulus size. Improving psychophysical thresholds with increasing size would indicate the presence of a mechanism that benefits from the integration of motion signals over space (i.e., spatial summation). In contrast, worsening performance with increasing size would indicate the presence of a mechanism that is negatively affected, or inhibited, by larger stimuli, a mechanism exhibiting spatial suppression.

\section{Global form discrimination experiment}

Subjects. A subset of the patients ( $n=10$; four females) also was tested on a global form task. The mean age of these patients was 38.3 years (SD, 7.8 years), mean education level was 12.6 years (SD, 2.02 years), and they had been ill for an average of 14.5 years (SD, 8.7 years).

Also tested on this form task were 15 healthy control subjects ( 9 females), recruited from the community, with no history of mental illness or neurological disorders. Their mean age was 36.6 years (SD, 11.8 years) and their mean education level was 13.5 years (SD, 2.3 years), values not significantly different from the patient group. Normal subjects were screened to rule out schizotypal personality using the SPQ (27) before the experiment. Mean score on the SPQ was 18.1 (SD, 11.1). No control subject was receiving psychotropic medications.

Psychophysical task. This four-alternative, forced-choice task measured the subject's ability to group small, stationary line elements into a larger, global form. The entire screen of the computer monitor was divided into four equal-sized quadrants whose boundaries were delineated by thick black lines, and the screen was filled with short lines, most of which were oriented randomly. Each line subtended a visual angle $\sim 30$ min length by 2 min width, and the lines appeared black against a gray background. In one of the four quadrants, a small group of six lines formed a quasicircular shape within a randomly selected region of the quadrant, and the probability of appearance in any of the quadrants was equal over the trials. To manipulate the clarity of the target, we intro-
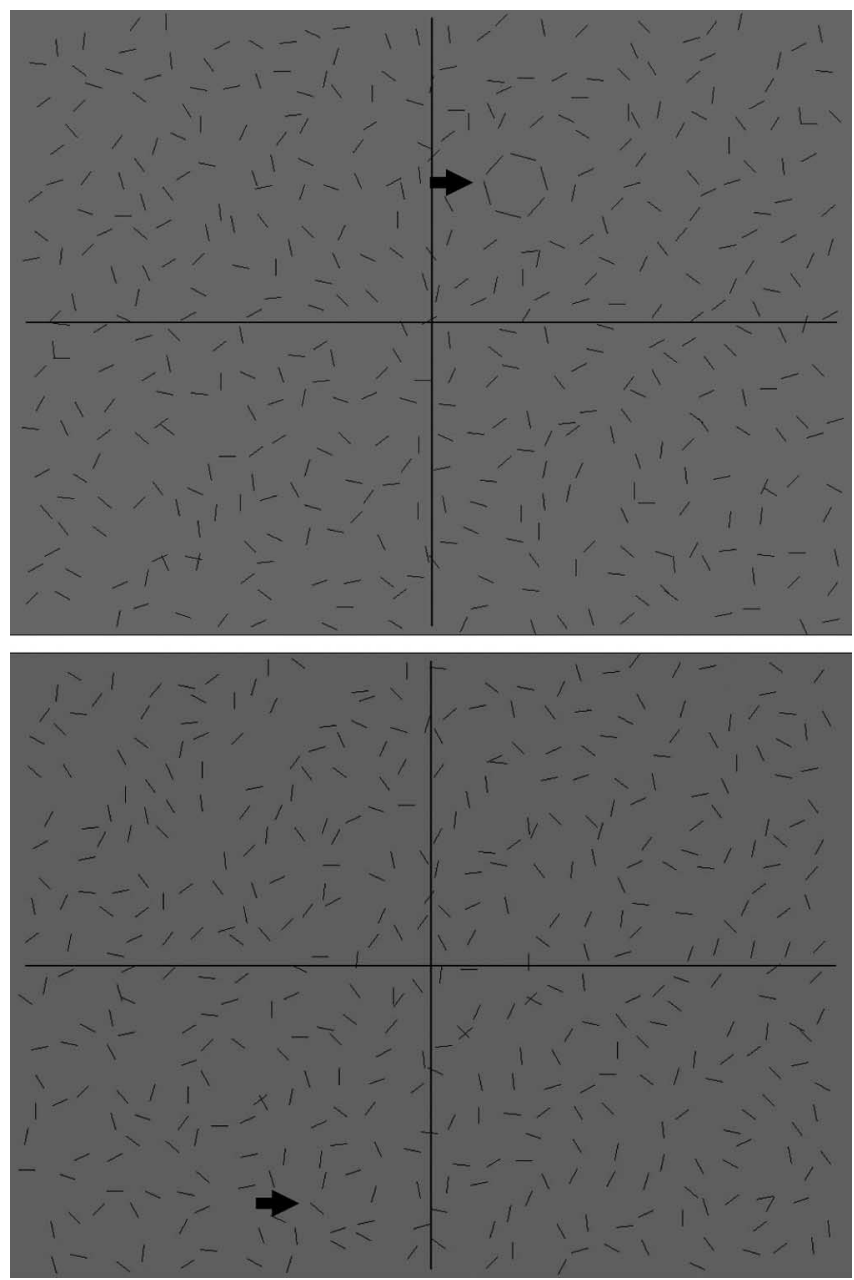

Figure 2. Examples of stimuli used for the global form task. The target was a quasicircular shape formed by six nearby lines. Task difficulty was adjusted by varying line jitter (the angular deviations among target contours from the canonical values). In the top example, there is no line jitter, and the target is easily detected (see arrow). The bottom example shows a difficult trial in which the target is hard to find because of substantial line jitter (see arrow).

duced "jitter" in the orientation of each line segment forming the quasicircular shape; jitter comprised an angular deviation among target contours from the canonical value specified by their positions on the circle. Therefore, larger degrees of jitter lessened the clarity of the target, resulting in impairment of subjects' ability to identify the quadrant in which the target appeared. Displays remained visible until the subject responded. Viewing distance was $57 \mathrm{~cm}$, and the visual angle of the target was $\sim 2.5^{\circ}$. Examples of the displays are shown in the Figure 2.

Each subject was instructed to locate the quasicircular shape that looked like "stop sign" and to indicate in which quadrant it appeared. Although no sample stimuli were presented before the formal testing, the test began with a series of trivially easy trials (jitter, 0 ), so that each subject quickly became accustomed to the task. The degree of jitter over trials was adjusted by a staircase procedure to find the level of jitter at which the subject could identify the target location with $>70 \%$ accuracy. Thus, the target became more difficult to detect after correct answers and less difficult after incorrect answers. Visual feedback showing correct location was provided after each trial. The total number of trials was 100, and the mean and SD of the jitter from the last 8 trials of the staircase were recorded as the estimate of the threshold. The subject could rest at any time during trials.

\section{Results}

When a moving stimulus was presented at low contrast, performance of both patients and controls improved as the stimulus 


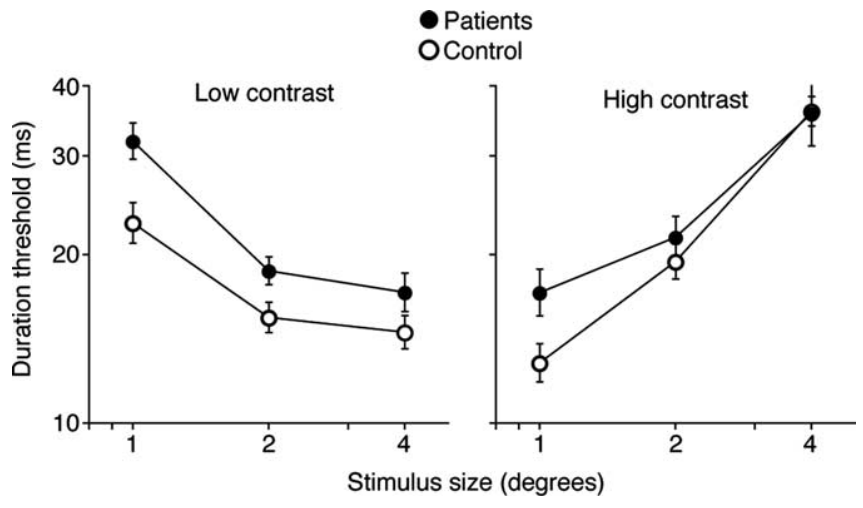

Figure 3. Effects of size and contrast on motion discrimination thresholds in patients and controls. The left panel shows the effects of stimulus size on the discriminability of low-contrast moving stimuli. The right panel depicts the effects increasing stimulus size has on high-contrast moving stimuli. Error bars are SEM.

size increased $\left(F_{(2,27)}=65.1 ; p<0.001\right)$ (Fig. 3, left panel). Patients' thresholds, however, were $\sim 25 \%$ higher than the control results $\left(F_{(1,28)}=7.4 ; p=0.01\right)$. The overall pattern of patient results closely mimicked the results for control subjects: as stimulus size increased, both patients and controls showed comparable improvements $\left(F_{(2,27)}=1.59 ; p=0.22\right)$. This pattern of results suggests unimpaired spatial summation mechanisms in motion perception. Uniform vertical shift of patients' thresholds can indicate either a deficit in perceiving low-contrast stimuli and/or a deficit in the perception of brief moving stimuli. Previous work has shown that contrast sensitivity for local motion is unimpaired in schizophrenia (Chen et al., 2003a). Chen et al. (2003a) also reported that patients are unimpaired at perceiving the motion of large, low contrast motions. Their stimuli, however, were presented for $300 \mathrm{~ms}$, which is an order of magnitude longer than duration thresholds measured for large moving stimuli in our experiment, suggesting that the observed deficit likely indicates an abnormality in the perception of brief motions. Indeed, a deficit in processing of brief visual stimuli has been associated with schizophrenia (Keri et al., 2005b).

At high contrast, performance of both patients and controls deteriorated as the stimulus size increased $\left(F_{(2,27)}=38 ; p<\right.$ 0.001) (Fig. 3, right panel). This pattern of results can be construed as an indicator of the presence of antagonistic centersurround interactions (Tadin et al., 2003). At high contrast, no main effects of group were found $\left(F_{(1,28)}=1.76 ; p=0.19\right)$. The only observed deficit was in a condition in which patients discriminated a small $\left(1^{\circ}\right)$ high-contrast motion $\left(t_{(28)}=2.29 ; p=\right.$ 0.03 ). The magnitude of the observed deficit was comparable with the threshold increases observed for low-contrast moving stimuli (Fig. 3). Importantly, as the stimulus size increased, the difference between patient and control data were eliminated. The interaction, however, was not significant $\left(F_{(2,27)}=1.72 ; p=\right.$ 0.19 ). This pattern of results might indicate that patients are selectively impaired in perceiving small moving objects. However, we find no evidence for such selective impairment in the lowcontrast results. Another possibility is that the relatively smaller threshold increase with increasing size reflects weaker centersurround suppression in schizophrenia. Specifically, a deficit in the perception of brief moving stimuli might be offset by weaker center-surround suppression. Center-surround suppression impairs motion perception only when the stimulus size is large, greater than the small stimuli used in the present study (Tadin et al., 2003). Thus, any "benefits" of reduced surround suppression would be apparent only at larger stimulus sizes.
We also compared patients' performance with low- and highcontrast moving stimuli. The analysis revealed that those patients who had lower thresholds for discriminating the motion of a small, low-contrast stimulus also had low thresholds for discriminating the motion of small, high-contrast stimuli $(r=0.52 ; p=$ $0.04)$. This, perhaps expected, observation simply indicates that patients who do better on one motion task tend do well in a related motion task. In contrast, doing well when the stimulus was a large, low-contrast stimulus does not predict patients' performance with large, high-contrast moving stimuli $(r=0.016$; $p=0.95)$. Control subjects showed a very similar pattern of results. This observation suggests that factors in addition to the general ability to perceive motion determine motion sensitivity for large, high-contrast stimuli. One such factor is the strength of surround suppression.

To quantify the strength of center-surround suppression at high contrast, we computed the "suppression index": $\log _{10}$ (threshold for the large, high-contrast stimulus) - $\log _{10}$ (threshold for the small, high-contrast stimulus). Analogously, to quantify the strength of spatial summation at low contrast, we computed the "summation index": $\log _{10}$ (threshold for the large, low-contrast stimulus) $-\log _{10}$ (threshold for the small, low-contrast stimulus). The suppression index is typically positive, because thresholds for large, high-contrast stimuli tend to be high relative to thresholds for small, low-contrast stimuli. In contrast, the summation index is typically negative, because thresholds for large, low-contrast stimuli tend to be low relative to thresholds for small, lowcontrast stimuli. Next, we compared suppression and summation index estimates with patient scores on scales used to assess clinical symptoms in schizophrenia: BPRS, SANS, and SAPS (Fig. $4)$. The summation index showed no dependency on symptom severity (all $r<0.36$; all $p>0.17$ ). The suppression index was not correlated with either BPRS $(r=-0.32 ; p=0.22)$ or SAPS $(r=0.1 ; p=0.71)$, but it was correlated with negative symptom severity (SANS) $(r=-0.54 ; p=0.03)$. Thus, patients with more severe negative symptoms exhibited weaker center-surround suppression. Moreover, from Figure 4, it is apparent that most patients (13 of 16) demonstrated center-surround suppression that was weaker than the average suppression measured for control subjects; and 10 of 16 patients had suppression indices lower than the lower quartile of the control data.

\section{Median split results}

\section{High contrast}

To further examine the relationship between symptom severity and high- and low-contrast results, we split patients into two groups relative to the median score on BPRS, SANS, and SAPS (Fig. $5 A$ ). At high contrast, we found an interaction when patients were split according to BPRS scores $\left(F_{(4,52)}=2.97 ; p=0.028\right)$, and there was a trend toward interaction when patients were split according to SANS scores $\left(F_{(4,52)}=2.40 ; p=0.06\right)$. No main effects of group were found (all $F_{(2,27)}<2.34$; all $p>0.12$ ). Patients with high BPRS and SANS scores tend to have higher thresholds for small and medium moving stimuli and slightly lower thresholds for the largest stimulus tested (the results for patients with low BPRS and SANS scores were essentially identical to control results). This tendency is also found when BPRS and SANS scores are correlated with duration thresholds (Fig. 5A, numbers under data symbols): positive correlations are found for small- and medium-size stimuli, and slight negative correlations were observed for large moving stimuli.

We performed an analogous three-group analysis on suppres- 

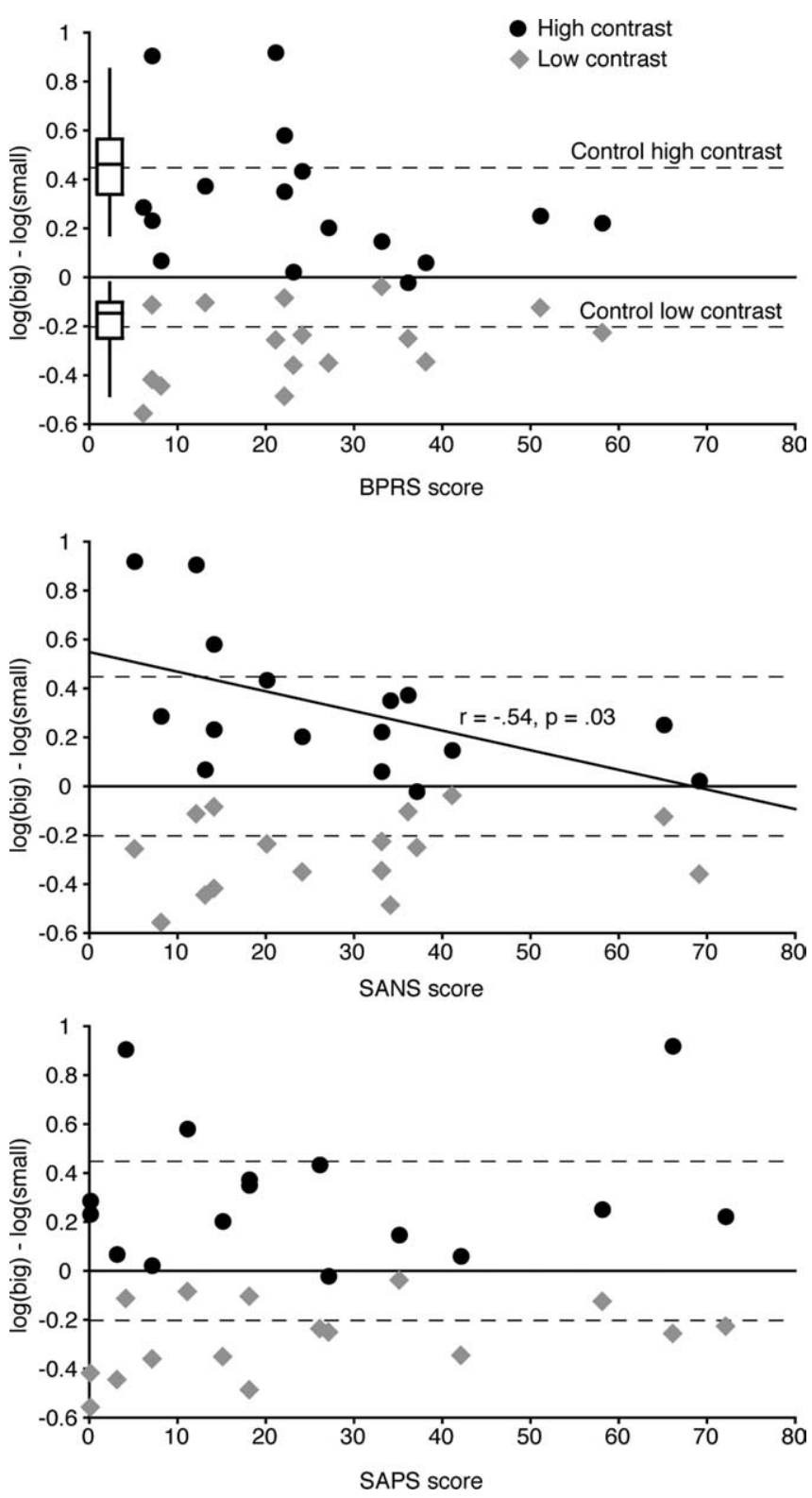

Figure 4. Relationship between the effect of stimulus size and symptom severity in schizophrenia. Positive $y$-axis values indicate that the psychophysical threshold for perceiving motion direction of the large stimulus was higher than that for perceiving motion direction of the small moving stimulus, a result suggesting surround suppression. Negative $y$-axis values indicate that motion of the large moving stimulus was easier to perceive than that of the small moving stimulus, a result suggesting spatial summation. The black circles depict high-contrast results for individual patients, whereas the gray diamonds show low-contrast results for each patient tested. The dashed lines in each panel show average high- and low-contrast results for control subjects. The box plots are added to better illustrate the range of the control results (with the middle horizontal line representing the median, the horizontal box boundaries representing the quartiles, and the "whiskers" stretching out to the minimum and the maximum values).

sion indices (Fig. 5B, light bars). Significant effects were found when patients were split according to BPRS $\left(F_{(2,27)}=5.29 ; p=\right.$ $0.012)$ and SANS scores $\left(F_{(2,27)}=4.57 ; p=0.02\right)$. No effect was found when patients were split according to SAPS scores $\left(F_{(2,27)}=\right.$ $1.09 ; p=0.35)$. This pattern of results indicates that patients with high BPRS and SANS scores exhibit weaker center-surround suppression than controls and patients with milder symptoms.

Finally, 4 of 16 patients exhibited no surround suppression: increasing stimulus size at high contrast had no effect on their thresholds (on average, their thresholds increased by $<2 \mathrm{~ms}$ ).
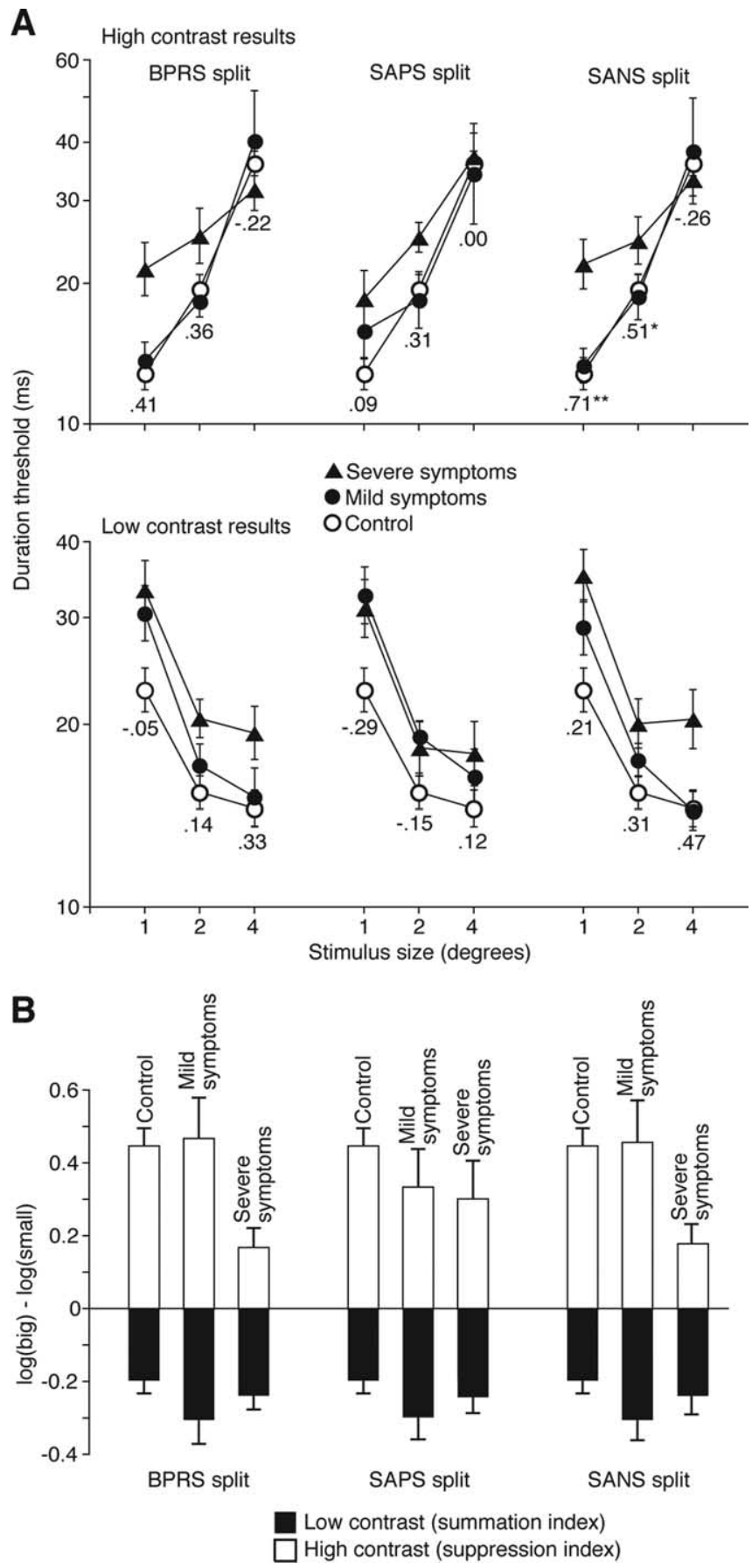

Figure 5. Median split analysis results. $A$, Duration thresholds as a function of stimulus size in control subjects and patients subgroups split around median BPRS, SAPS, and SANS scores. The top and bottom panels show results from high- and low-contrast conditions, respectively. The numbers under data symbols indicate correlations between BPRS, SAPS, and SANS scores and duration thresholds for given stimulus size. For example, the leftmost number in the top panel $(r=0.41)$ indicates the correlation between BPRS scores and duration thresholds for small, high-contrast moving stimuli. Error bars indicate SEM. $\boldsymbol{B}$, Effect of stimulus size on motion perception of control subjects and patients subgroups split around median BPRS, SAPS, and SANS scores. The $y$-axis convention is the same as in Figure 4 , in which higher positive numbers indicate strong center-surround suppression and negative numbers indicate spatial summation.

Similar to other patients, this subgroup had elevated duration thresholds when the moving stimulus was small (13 ms threshold increase; $t_{(16)}=3.48 ; p=0.003$ ). However, when the stimulus was large, patients in this subgroup exhibited thresholds $30 \%$ 
lower than control subjects' thresholds ( 9 ms threshold decrease; $\left.t_{(16)}=2.25 ; p=0.039\right)$. This indicates that a lack of centersurround suppression can result in reduced thresholds for perceiving large, high-contrast motion. Moreover, this threshold reduction can reach levels where patients are actually outperforming control subjects. We examined clinical profiles of these four patents, but we found no significant difference from the rest of the schizophrenic subjects. The small number of patients with no surround suppression, however, limits our ability to discern small but real differences between two groups.

\section{Low contrast}

At low contrast (Fig. 5A), schizophrenic psychosis was associated with higher duration thresholds (all group $F_{(2,27)}>3.58$; all $p<$ $0.04)$. A trend toward significant interaction was found when patients were split according to SANS scores $\left(F_{(4,52)}=2.40 ; p=\right.$ $0.06)$. However, an analogous three-group analysis on summation indices (Fig. 5B, dark bars) yielded no differences between groups (all $F_{(2,27)}<1.35$; all $\left.p>0.28\right)$. Thus, the overall pattern of results was similar across different groups except for vertical shifts indicated by significant group differences. To further examine the differences between patients with severe and mild symptoms, we excluded control subjects from the analysis of duration thresholds. The effect of group was significant when patients were split according to SANS scores $\left(F_{(1,14)}=4.6 ; p=\right.$ $0.05)$, and failed to reach significance when patients were split according to BPRS and SAPS scores (all $F_{(1,14)}<2.24$; all $p>$ $0.15)$.

Overall, both the correlation and the median split analyses revealed an inverse relationship between severity of negative symptoms in schizophrenia and the strength of center-surround suppression at high contrast. Center-surround suppression in control subjects was over 2.5 times stronger than centersurround suppression estimated for patients with severe negative symptoms (Fig. 5B).

\section{Medication}

It is important to consider possible effects of medication. For example, there is evidence suggesting that dopamine antagonists affect visual contrast sensitivity (Chen et al., 2003b). Dopamine is critical for the ability to detect visual contrast, and antipsychotic drugs block dopamine receptors. The newer, "atypical" antipsychotics, such as risperidone or olanzapine, block dopamine receptors for much shorter periods than typical neuroleptic drugs such as haloperidol. Indeed, Chen et al. (2003b) found that the thresholds for detecting visual contrast in schizophrenia patients taking typical antipsychotic drugs were elevated. In contrast, the thresholds for those who were taking atypical antipsychotics were the same as that found in healthy subjects. All of our patients were receiving atypical antipsychotic drugs; thus, we did not expect to find perceptual deficits associated with typical antipsychotics.

Indeed, we found no correlation between $\mathrm{CPZ}$ dose and suppression and summation indices (all $r<0.32$; all $p>0.23$ ). We also examined whether medication dose for four patients that exhibited no surround suppression differed from the rest of schizophrenic patients, but we did not find any differences $\left(t_{(14)}=\right.$ $0.15 ; p=0.88)$. Moreover, patients' thresholds on different motion tasks did not correlate with the CPZ doze. Highest correlation was found between $\mathrm{CPZ}$ and thresholds for the big, highcontrast stimuli $(r=-0.46 ; p=0.08)$. All other correlations were $<0.20$. Finally, there was no relationship between CPZ dose and symptom severity as measured by BPRS, SANS, and SAPS (all $r<0.40$; all $p>0.13$ ).
It has been hypothesized that weakened surround suppression might be caused by an abnormal GABAergic system (Betts et al., 2005). Indeed, GABAergic effects on visual perception (Blin et al., 1993) and motion integration and segmentation (Giersch and Lorenceau, 1999) have been reported. Thus, use of medication that has considerable effects on the GABAergic system might lead to unwanted confounds. However, atypical antipsychotic drugs that our patients were taking mostly work on dopamine receptors and, to a lesser degree, on serotonergic receptors. Thus, any effects on the GABAergic system are indirect and likely minor.

\section{Global form discrimination experiment}

The results presented so far demonstrate a motion-processing deficit in schizophrenia. It is, however, useful to examine performance of schizophrenic patients in a nonmotion visual task. An unimpaired performance on another visual task would suggest that these patients do not exhibit a general visual perception deficit. Moreover, psychophysical experiments are relatively tedious and sustaining motivation over the course of an experiment can be difficult. Thus, it is important to establish that patients can perform within the normal range in a comparable psychophysical task.

To perform these necessary controls, we measured patients' perceptual ability to group small, stationary line elements into a larger, global form (Fig. 2). The mean jitter threshold values (the range of angular deviations among line elements) for patients did not differ from control data $\left(t_{(23)}=0.807 ; p=0.43\right)$. This result essentially replicates a previous finding from our laboratory (Kim et al., 2005) and demonstrates that schizophrenic patients can perform in a normal range on a nonmotion perceptual task. Performance of patients on the global form task did not correlate with overall, positive, nor negative symptoms. These results provide additional evidence that visual processing in schizophrenia is relatively intact in visual tasks requiring processing of detailed form information (O’Donnell et al., 1996, 2002).

\section{Discussion}

We show that schizophrenic patients have elevated direction discrimination thresholds while performing normally in a shape discrimination task. This result provides additional evidence for the existence of a motion processing deficit in schizophrenia (Stuve et al., 1997; Chen et al., 1999a,b,c, 2003a, 2004, 2005; Li, 2002; Slaghuis et al., 2005; Kim et al., 2006). More importantly, at high contrast, schizophrenic patients exhibit reduced centersurround suppression. Because of this reduction in estimated surround suppression, the average thresholds of schizophrenic subjects for the largest stimulus size were the same as those for the controls (Fig. 3). In fact, four patients showed no effect of stimulus size at high contrast, a finding that we have yet to observe in normal, age-matched subjects. Those patients also had lower thresholds (better performance) than control subjects for perceiving large high-contrast moving stimuli. This counterintuitive observation is likely caused by weakened suppressive mechanisms in motion processing, mechanisms that normally yield elevated thresholds for perceiving large, high-contrast motion in healthy young subjects.

At low contrast, changes in stimulus size have essentially the same effect for schizophrenic and control subjects. Thus, the main qualitative difference between schizophrenic patients and control subjects is found at high contrast, where increases in the stimulus size had a much larger effect in motion perception of control subjects. In other words, the low contrast condition can 
also be considered a control condition for the general effect of stimulus size.

\section{Possible functional consequences of weakened surround suppression}

Lesser impairment in motion discrimination with increasing stimulus size observed in schizophrenia, however, should not be considered a perceptual advantage, because it indicates weaker surround suppression. Suppressive center-surround interactions have been implicated in a variety of perceptual functions (Tadin and Lappin, 2005). In motion perception, center-surround interactions have been linked with the segmentation of moving objects from the background (Nakayama and Loomis, 1974; Allman et al., 1985; Born et al., 2000), slow visual pursuit of moving objects (Born et al., 2000) and perception of their three-dimensional shape (Xiao et al., 1995; Buracas and Albright, 1996). Abnormality in surround suppression may impair these important perceptual functions. Indeed, schizophrenic patients have trouble segmenting moving forms from the background (Schwartz et al., 1999; Kim et al., 2005).

\section{Comparison with other contextual processing deficits}

Center-surround interactions are a type of contextual modulation in which visual stimulation of the surround modulates the response in the center region. Our report of abnormal centersurround interactions in motion perception is consistent with other studies reporting abnormal contextual interactions in schizophrenia (Must et al., 2004; Dakin et al., 2005; Keri et al., 2005a,b). In some cases, contextual interactions can affect the appearance of visual stimuli (e.g., contrast) (Chubb et al., 1989), implying that, in those cases, abnormally weak contextual modulations might counterintuitively yield a veridical perception of the affected stimulus property. Indeed, schizophrenic patients are more accurate at perceiving stimulus contrast under contextual manipulations that typically affect contrast perception of control subjects (Dakin et al., 2005). Moreover, Keri and colleagues found that weakening of contextual interactions in schizophrenia was correlated with negative symptom severity in one study (Keri et al., 2005b), but not in two related investigations (Must et al., 2004; Keri et al., 2005a). Testing of highfunctioning patients (mean SANS, $<10$ ), however, likely reduced the chances of finding a significant correlation.

\section{Potential causes of abnormal center-surround interactions in schizophrenia}

Psychophysically observed center-surround interactions in motion perception have been linked with center-surround antagonism in cortical area MT (Tadin et al., 2003). Specifically, the dependency of psychophysical surround suppression on contrast, eccentricity, stimulus isoluminance, and motion adaptation is consistent with what is known about response properties of neurons in MT. Moreover, our medium and large stimuli are, respectively, large enough to partially and substantially stimulate the surrounds of foveal MT receptive fields (for details, see Materials and Methods). Thus, abnormally weak surround suppression in schizophrenia suggests the existence of a deficit in MT processing. Other research has shown that schizophrenic patients are deficient in perceiving random-dot motion (Chen et al., 2003a), which is a task that is also linked with MT neurons (Salzman et al., 1990). Likewise, an impairment of the later stages of motion processing is suggested by the observation that velocity discrimination deficits in schizophrenia are independent of contrast (Chen at al., 2004). Furthermore, a recent functional mag- netic resonance imaging study reported an abnormal MT activity while schizophrenic patients performed a SPEM task (Lencer et al., 2005), a task that is affected by the activity of center-surround neurons in MT (Born et al., 2000). We cannot rule out a contribution from V1 and other motion sensitive areas, of course. Indeed, suppressive center-surround receptive-field interactions are found in V1 (Jones et al., 2001), albeit at a much smaller spatial scale.

What particular abnormality might be responsible for weak surround suppression in schizophrenia? A similar pattern of results as in the present study was found in elderly subjects (Betts et al., 2005). In fact, because of reduced surround suppression, elderly subjects significantly outperformed young subjects in motion perception of large high-contrast patterns. Age-related decrease in GABA-mediated inhibition has been associated with perceptual deficits in primates (Schmolesky et al., 2000; Leventhal et al., 2003). This led Betts et al. (2005) to suggest that decreased cortical inhibition is responsible for the weakening of inhibitory center-surround interactions and, thus, for improved motion perception of large high-contrast patterns. A deficit in the GABAergic system is well documented in schizophrenia (Wassef et al., 2003), which suggests a possibility that similar deficits in the GABA system may underlie the abnormality in surround suppression for both schizophrenic and elderly subjects (for medication-related issues, see Results). Furthermore, similar velocity discrimination deficits exist in elderly and schizophrenic subjects (Bidwell et al., 2006). Elderly and schizophrenic subjects also exhibit comparable impairments in the perception of motion-defined forms (Schwartz et al., 1999; Wist et al., 2000), a task that is likely facilitated by unimpaired center-surround suppression mechanisms (Allman et al., 1985; Tadin and Lappin, 2005). Moreover, elderly subjects show SPEM abnormalities (Morrow and Sharpe, 1993; Knox et al., 2005), which nicely dovetails with a well documented SPEM deficit in schizophrenia (Holzman et al., 1973). These correspondences between schizophrenia- and age-related defects suggest a general similarity and/or overlap in underlying motion-processing deficits in two populations.

\section{Negative symptoms}

The weakening of center-surround suppression in schizophrenia was correlated with the negative symptom severity, as measured by SANS (Figs. 4, 5). Growing evidence suggests that visual perception deficits in schizophrenic patients are typically associated with negative symptoms rather than positive symptoms (Cadenhead et al., 1997; Slaghuis and Bishop, 2001; Butler et al., 2003; Slaghuis, 2004; Keri et al., 2005b). Negative symptoms in turn are linked with reduced social functioning and poor outcome. Thus, abnormal visual information processing may be a factor in impoverished social functioning (Sergi and Green, 2003; Sergi et al., 2006) and may hinder successful rehabilitation. Humans depend heavily on vision for understanding and interacting with the world around them. Deficits in visual perception, especially in accurately perceiving dynamic, moving stimuli may result in disadvantages of social perception.

The link between negative symptoms and weaker surround suppression is perhaps counterintuitive. However, suppression at one level does not necessarily imply suppression at another. For example, schizophrenia is associated with reduced frontal metabolism. Hypofrontality in schizophrenia seems to be directly related to disinhibition of attention and oculomotor behavior (Levin, 1984) as well as striatal dopaminergic function (MeyerLindenberg et al., 2002). Moreover, hypofrontality is linked with 


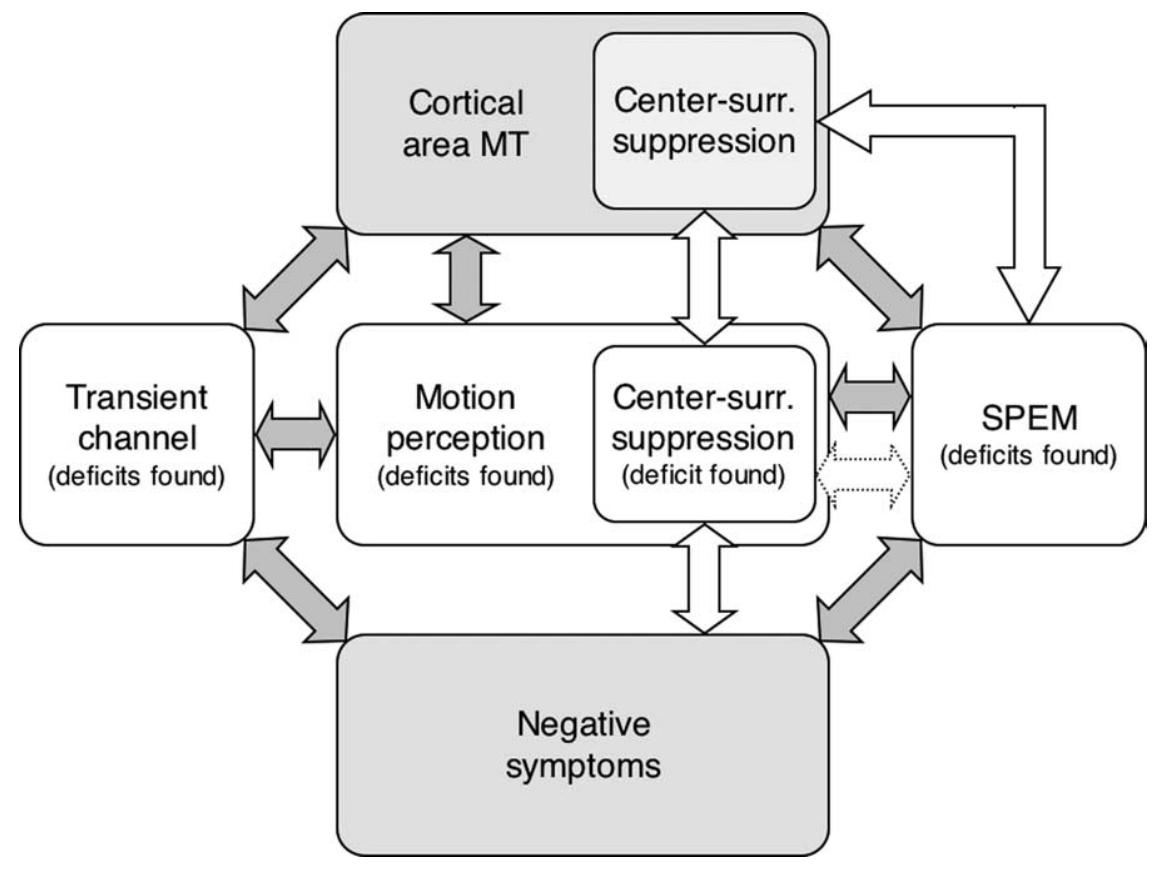

Figure 6. Transient channel perceptual deficits in schizophrenia. The presented framework is intended as an overview of the main trends in the literature and not as a comprehensive model. Some of the presented links pertain to the experimental questions that are still actively investigated (e.g., a link between negative symptoms and SPEM), and thus, there is a possibility that those links might need to be altered or perhaps even eliminated. Area MT links (top row of arrows), Numerous neurophysiological, psychophysical, and clinical results link cortical area MT with motion perception, the transient (magnocellular) channel, and center-surround suppression of moving stimuli (Orban, 1997; Born and Bradley, 2005). Moreover, neural processing within area MT is critically involved in SPEM (Wurtz et al., 1990). Thus, deficits in these perceptual and oculomotor motor functions suggest a possible MT deficit in schizophrenia. Links among perceptual and oculomotor deficits (horizontal arrows), Motion perception is one of the key functions of the transient channel. Thus, nearly all motion perception deficits can be considered transient channel deficits. Motion perception is also a critical first step in SPEM generation (Ilg, 1997). For example, the magnitude of motion perception deficits in schizophrenia predicts the severity of the SPEM deficit, suggesting a link between SPEM and motion perception deficits (Stuve et al., 1997; Chen et al., 1999b,c). More research is required to elucidate the connections among various perceptual deficits in schizophrenia and to test a possible perceptual origin of the SPEM deficit. Links between negative symptoms and perceptual/oculomotor abnormalities in schizophrenia (bottom row of arrows), Several studies have found that patients with predominantly negative symptoms have more pronounced transient channel deficits (Cadenhead et al., 1997; Slaghuis and Bishop, 2001; Butler et al., 2003). Moreover, a connection between negative or deficit symptoms and SPEM deficit has been found with a variety of experimental designs (Sweeney et al., 1994; Ross et al., 1996, 1997; Roitman et al., 1997; Slaghuis et al., 2005) (but see Kelly et al., 1990; Nkam et al., 2001). There is, however, an inherent difficulty in comparing a stable, trait-like characteristic such as SPEM defect and clinical symptom severity that varies over time. Therefore, depending on when patients are examined, correlation between symptoms and SPEM deficit may or may not be observed. What is relevant for our purpose is that a large majority of studies that examined the relationship between clinical symptoms and SPEM found a link with negative symptoms. The existence of a link between negative symptoms and motion perception deficits (e.g., velocity discrimination, perception of random-dot motion) has yet to be fully investigated. Nevertheless, based on the central role of motion perception in the transient visual channel, we suspect that such a connection exists. Center-surround suppression links (white arrows), The remaining connections pertain to the results of this paper. The task used in the present study is believed to reflect cortical center-surround mechanisms, particularly those within area MT (Tadin et al., 2003). The activity of MT center-surround neurons has been shown to directly affect SPEM (Born et al., 2000), suggesting a functional link between SPEM and surround suppression. The remaining full arrow depicts the link between surround suppression and negative symptoms (Figs. 4, 5). The present finding suggests the possibility of a connection between center-surround suppression abnormality in schizophrenia and SPEM deficit (dotted arrow). Such a link is consistent with other connections shown in the figure.

negative symptoms in medicated (Wolkin et al., 1992; Lahti et al., 2001) and neuoleptic-naive (Andreasen et al., 1997) patients. Thus, weakened frontal cortical control over cortical and subcortical circuits seems to lie at the heart of negative symptomatology. In other words, disinhibition (i.e., disinhibited dopamine system) at one level is linked to outward appearance of behavioral suppression.

As outlined in Introduction, a large number of visual defects in schizophrenia tend to involve perception and visual pursuit of dynamic and moving stimuli. Many of these processes have been linked with the magnocellular visual processing stream, and as- sociated with negative symptoms. To present our findings within the context of these results, we prepared a simple schematic framework that outlines and summarizes the links among relevant perceptual deficits, area MT, and clinical symptoms (Fig. 6). This schematic diagram highlights the interrelationship between perception and symptoms and proposes a framework for additional hypothesis testing. In any case, it is apparent that many perceptual deficits associated with schizophrenia share several characteristics, an observation suggesting a common factor(s) behind visual abnormalities in schizophrenia.

In summary, we show that schizophrenic patients show abnormally weak center-surround interactions in motion perception. This deficit was most pronounced in patients with severe negative symptoms. Interestingly, patients with the weakest surround suppression actually outperformed control subjects in motion discriminations of large high-contrast stimuli. This enhanced motion perception of large high-contrast stimuli is consistent with an MT abnormality and has a potential to disrupt functions that depend on unimpaired center-surround suppression in motion.

\section{References}

Albright TD, Desimone R (1987) Local precision of visuotopic organization in the middle temporal area (MT) of the macaque. Exp Brain Res 65:582-592.

Allman J, Meizin F, McGuiness E (1985) Direction- and velocity-specific responses from beyond the classical receptive field in the middle temporal visual area (MT). Perception 14:105-126.

Andreasen NC, Olsen S (1982) Negative v positive schizophrenia: definition and validation. Arch Gen Psychiatry 39:789-794.

Andreasen NC, O’Leary DS, Flaum M, Nopoulos P, Watkins GL, Boles Ponto LL, Hichwa RD (1997) Hypofrontality in schizophrenia: distributed dysfunctional circuits in neurolepticnaive patients. Lancet 349:1730-1734.

Betts LR, Taylor CP, Sekuler AB, Bennett PJ (2005) Aging reduces center-surround antagonism in visual motion processing. Neuron 45:361-366.

Bidwell LC, Holzman PS, Chen Y 2006 Aging and visual motion discrimination in normal adults and schizophrenia patients. Psychiatry Res, in press.

Blin O, Mestre D, Paut O, Vercher JL, Audebert C (1993) GABA-ergic control of visual perception in healthy volunteers: effects of midazolam, a benzodiazepine, on spatio-temporal contrast sensitivity. Br J Clin Pharmacol 36:117-124.

Born RT, Bradley DC (2005) Structure and function of visual area MT. Annu Rev Neurosci 28:157-189.

Born RT, Tootell RB (1992) Segregation of global and local motion processing in primate middle temporal visual area. Nature 357:497-499.

Born RT, Groh JM, Zhao R, Lukasewycz SJ (2000) Segregation of object and background motion in visual area MT: effects of microstimulation on eye movements. Neuron 26:725-734. 
Brainard DH (1997) The psychophysics toolbox. Spat Vis 10:433-436.

Buracas GT, Albright TD (1996) Contribution of area MT to perception of three-dimensional shape: computational study. Vision Res 361:869-887.

Butler PD, Javitt DC (2005) Early-stage visual processing deficits in schizophrenia. Curr Opin Psychiatry 18:151-157.

Butler PD, DeSanti LA, Maddox J, Harkavy-Friedman JM, Amador XF, Goetz RR, Javitt DC, Gorman JM (2003) Visual backward-masking deficits in schizophrenia: relationship to visual pathway function and symptomatology. Schizophr Res 59:199-209.

Cadenhead KS, Geyer MA, Butler RW, Perry W, Sprock J, Braff DL (1997) Information processing deficits of schizophrenia patients: relationship to clinical ratings, gender and medication status. Schizophr Res 28:51-62.

Chen Y, Palafox GP, Nakayama K, Levy DL, Matthysse S, Holzman PS (1999a) Motion perception in schizophrenia. Arch Gen Psychiatry 56:149-154.

Chen Y, Levy DL, Nakayama K, Matthysse S, Palafox G, Holzman PS (1999b) Dependence of impaired eye tracking on deficient velocity discrimination in schizophrenia. Arch Gen Psychiatry 56:155-161.

Chen Y, Nakayama K, Levy DL, Matthysse S, Holzman PS (1999c) Psychophysical isolation of a motion-processing deficit in schizophrenics and their relatives and its association with impaired smooth pursuit. Proc Natl Acad Sci USA 96:4724-4729.

Chen Y, Nakayama K, Levy D, Matthysse S, Holzman P (2003a) Processing of global, but not local, motion direction is deficient in schizophrenia. Schizophr Res 61:215-227.

Chen Y, Levy DL, Sheremata S, Nakayama K, Matthysse S, Holzman PS (2003b) Effects of typical, atypical, and no antipsychotic drugs on visual contrast detection in schizophrenia. Am J Psychiatry 160:1795-1801.

Chen Y, Levy DL, Sheremata S, Holzman PS (2004) Compromised latestage motion processing in schizophrenia. Biol Psychiatry 55:834-841.

Chen Y, Bidwell LC, Holzman PS (2005) Visual motion integration in schizophrenia patients, their first-degree relatives, and patients with bipolar disorder. Schizophr Res 74:271-281.

Chubb C, Sperling G, Solomon JA (1989) Texture interactions determine perceived contrast. Proc Natl Acad Sci USA 86:9631-9635.

Dakin S, Carlin P, Hemsley D (2005) Weak suppression of visual context in chronic schizophrenia. Curr Biol 15:R822-R824.

Gattass R, Gross CG (1981) Visual topography of striate projection zone (MT) in posterior superior temporal sulcus of the macaque. J Neurophysiol 46:621-638.

Giersch A, Lorenceau J (1999) Effects of a benzodiazepine, lorazepam, on motion integration and segmentation. Vision Res 39:2017-2025.

Gold JI, Shadlen MN (2000) Representation of a perceptual decision in developing oculomotor commands. Nature 404:390-394.

Green MF, Walker E (1986) Symptom correlates of vulnerability to backward masking in schizophrenia. Am J Psychiatry 143:181-186.

Holzman PS, Proctor LR, Hughes DW (1973) Eye-tracking patterns in schizophrenia. Science 181:179-181.

Ilg UJ (1997) Slow eye movements. Prog Neurobiol 53:293-329.

Jones HE, Grieve KL, Wang W, Sillito AM (2001) Surround suppression in primate V1. J Neurophysiol 86:2011-2028.

Kastner S, De Weerd P, Pinsk MA, Elizondo MI, Desimone R, Ungerleider LG (2001) Modulation of sensory suppression: implications for receptive field sizes in the human visual cortex. J Neurophysiol 86:1398-1411.

Kelly P, Rennie C, Gordon E, Anderson J, Howson A, Meares R (1990) Smooth pursuit eye tracking dysfunction and negative symptoms in schizophrenia. Psychiatr Res 34:89-97.

Keri S, Kelemen O, Benedek G, Janka Z (2005a) Lateral interactions in the visual cortex of patients with schizophrenia and bipolar disorder. Psychol Med 35:1043-1051.

Keri S, Kiss I, Kelemen O, Benedek G, Janka Z (2005b) Anomalous visual experiences, negative symptoms, perceptual organization and the magnocellular pathway in schizophrenia: a shared construct? Psychol Med 35:1445-1455.

Kim D, Wylie G, Pasternak R, Butler PD, Javitt DC (2006) Magnocellular contributions to impaired motion processing in schizophrenia. Schizophr Res 82:1-8.

Kim J, Doop ML, Blake R, Park S (2005) Impaired visual recognition of biological motion in schizophrenia. Schizophr Res 77:299-307.

Knox PC, Davidson JH, Anderson D (2005) Age-related changes in smooth pursuit initiation. Exp Brain Res 165:1-7.

Lahti AC, Holcomb HH, Medoff DR, Weiler MA, Tamminga CA, Carpenter
WT (2001) Abnormal patterns of regional cerebral blood flow in schizophrenia with primary negative symptoms during an effortful auditory recognition task. Am J Psychiatry 158:1797-1808.

Lencer R, Nagel M, Sprenger A, Heide W, Binkofski F (2005) Reduced neuronal activity in the V5 complex underlies smooth-pursuit deficit in schizophrenia: evidence from an fMRI study. NeuroImage 24:1256-1259.

Leventhal AG, Wang Y, Pu M, Zhou Y, Ma Y (2003) GABA and its agonists improved visual cortical function in senescent monkeys. Science 300:812-815.

Levin S (1984) Frontal lobe dysfunctions in schizophrenia-I. Eye movement impairments. J Psychiatr Res 18:27-55.

Li CS (2002) Impaired detection of visual motion in schizophrenia patients. Prog Neuropsychopharmacol Biol Psychiatry 26:929-934.

Meyer-Lindenberg A, Miletich RS, Kohn PD, Esposito G, Carson RE, Quarantelli M, Weinberger DR, Berman KF (2002) Reduced prefrontal activity predicts exaggerated striatal dopaminergic function in schizophrenia. Nat Neurosci 5:267-271.

Morrow MJ, Sharpe JA (1993) Smooth pursuit initiation in young and elderly subjects. Vision Res 33:203-210.

Must A, Janka Z, Benedek G, Keri S (2004) Reduced facilitation effect of collinear flankers on contrast detection reveals impaired lateral connectivity in the visual cortex of schizophrenia patients. Neurosci Lett 357:131-134.

Nakayama K, Loomis JM (1974) Optical velocity patterns, velocity-sensitive neurons, and space perception: a hypothesis. Perception 3:63-80.

Nkam I, Thibaut F, Denise P, Van Der Elst A, Segard L, Brazo P, Menard JF, Thery S, Halbeck I, Delamilleure P, Vasse T, Etard O, Dollfus S, Champion D, Levillian D, Petit M (2001) Saccadic and smooth-pursuit eye movements in deficit and nondefcit schizophrenia. Schizophr Res 48:145-153.

O'Donnell BF, Swearer JM, Smith LT, Nestor PG, Shenton ME, McCarley RW (1996) Selective deficits in visual perception and recognition in schizophrenia. Am J Psychiatry 153:687-692.

O'Donnell BF, Potts GF, Nestor PG, Stylianopoulos KC, Shenton ME, McCarley RW (2002) Spatial frequency discrimination in schizophrenia. J Abnorm Psychol 111:620-625.

Orban GA (1997) Visual processing in macaque area MT/V5 and its satellites (MSTd and MSTv). In Cerebral cortex, Vol 12 (Rockland KS, Kaas JH, Peters A, eds), pp 359-434. New York: Plenum.

Overall JE, Gorham DR (1962) The brief psychiatric rating scale. Psychol Rep 10:799-812.

Pack CC, Hunter JN, Born RT (2005) Contrast dependence of suppressive influences in cortical area MT of alert macaque. J Neurophysiol 93:1809-1815.

Paffen CL, Tadin D, Te Pas SF, Blake R, Verstraten FA (2006) Adaptive center-surround interactions in human vision revealed during binocular rivalry. Vision Res 46:599-604.

Pelli DG (1997) The video toolbox software for visual psychophysics: transforming numbers into movies. Spat Vis 10:437-442.

Raiguel SE, van Hulle MM, Xiao DK, Marcar VL, Orban GA (1995) Shape and spatial distribution of receptive fields and antagonistic motion surround in the middle temporal area (V5) of the macaque. Eur J Neurosci 7:2064-2082.

Raine A (1991) The SPQ: a scale for the assessment of schizotypal personality based on DSM III-R criteria. Schizophr Bull 17:556-564.

Ransil BJ, Schachter SC (1994) Test-retest reliability of the Edinburgh Handedness Inventory and Global Handedness preference measurements, and their correlation. Percept Mot Skills 79:1355-1372.

Rees G, Friston K, Koch C (2000) A direct quantitative relationship between the functional properties of human and macaque V5. Nat Neurosci 3:716-723.

Roitman JD, Shadlen MN (2002) Response of neurons in the lateral intraparietal area during a combined visual discrimination reaction time task. J Neurosci 22:9475-9489.

Roitman SE, Keefe RSE, Harvey PD, Siever LJ, Mohs RC (1997) Attentional and eye tracking deficits correlate with negative symptoms in schizophrenia. Schizophr Res 26:139-146.

Ross DE, Thaker GK, Buchanan RW, Lahti AC, Medoff D, Bartko JJ, Moran M, Hartley J (1996) Association of abnormal smooth-pursuit eye movements with the deficit syndrome in schizophrenic patients. Am J Psychiatry 153:1158-1165.

Ross DE, Thaker GK, Buchanan RW, Kirkpatrick B, Lahti AC, Medoff D, 
Bartko JJ, Goodman J, Tien A (1997) Eye tracking disorder in schizophrenia is characterised by specific oculomotor defects and is associated with the defcit syndrome. Biol Psychiatry 42:781-796.

Salzman CD, Britten KH, Newsome WT (1990) Cortical microstimulation influences perceptual judgements of motion direction. Nature 346:174-177.

Schechter I, Butler PD, Silipo G, Zemon V, Javitt DC (2003) Magnocellular and parvocellular contributions to backward masking dysfunction in schizophrenia. Schizophr Res 64:91-101.

Schmolesky MT, Wang Y, Pu M, Leventhal AG (2000) Degradation of stimulus selectivity of visual cortical cells in senescent rhesus monkeys. Nat Neurosci 3:384-390.

Schwartz BD, Maron BA, Evans WJ, Winstead DK (1999) High velocity transient visual processing deficits diminish ability of patients with schizophrenia to recognize objects. Neuropsychiatry Neuropsychol Behav Neurol 12:170-177.

Sergi MJ, Green MF (2003) Social perception and early visual processing in schizophrenia. Schizophr Res 59:233-241.

Sergi MJ, Rassovsky Y, Nuechterlein KH, Green MF (2006) Social perception as a mediator of the influence of early visual processing on functional status in schizophrenia. Am J Psychiatry 163:448-454.

Slaghuis WL (2004) Spatio-temporal luminance contrast sensitivity and visual backward masking in schizophrenia. Exp Brain Res 156:196-211.

Slaghuis WL, Bishop AM (2001) Luminance flicker sensitivity in positiveand negative-symptom schizophrenia. Exp Brain Res 138:88-99.

Slaghuis WL, Bowling AC, French RV (2005) Smooth-pursuit eye movement and directional motion-contrast sensitivity in schizophrenia. Exp Brain Res 166:89-101.

Spitzer RL, Williams JDW (1985) Structured clinical interview for DSM III-R. New York: New York State Psychiatric Institute Biomedical Research Division.

Spreen O, Strauss E (1998) A compendium of neuropsychological tests. New York: Oxford UP.
Stuve TA, Friedman L, Jesberger JA, Gilmore GC, Strauss ME, Meltzer HY (1997) The relationship between smooth pursuit performance, motion perception and sustained visual attention in patients with schizophrenia and normal controls. Psychol Med 27:143-152.

Sweeney JA, Clementz BA, Haas GL, Escobar MD, Drake K, Francis AJ (1994) Eye tracking dysfunction in schizophrenia: characterisation of component eye movement abnormalities, diagnostic specifcity and the role of attention. J Abnorm Psychol 103:222-230.

Tadin D, Lappin JS (2005) Linking psychophysics and physiology of centersurround interactions in visual motion processing. In: Seeing spatial form (Jenkin RM, Harris LR, eds), pp 279-314. Oxford: Oxford UP.

Tadin D, Lappin JS, Gilroy L, Blake R (2003) Perceptual consequences of centre-surround antagonism in visual motion processing. Nature 424:312-315.

Wassef A, Baker J, Kochan LD (2003) GABA and schizophrenia: a review of basic science and clinical studies. J Clin Psychopharmacol 23:601-640.

Watson AB, Pelli DG (1983) QUEST: a Bayesian adaptive psychometric method. Percept Psychophys 33:113-120.

Wechsler D (1981) WAIS-R manual. New York: The Psychological Corporation.

Wist ER, Schrauf M, Ehrenstein WH (2000) Dynamic vision based on motion-contrast: changes with age in adults. Exp Brain Res 134:295-300.

Wolkin A, Sanfilipo M, Wolf AP, Angrist B, Brodie JD, Rotrosen J (1992) Negative symptoms and hypofrontality in chronic schizophrenia. Arch Gen Psychiatry 49:959-965.

Wurtz RH, Yamasaki DS, Duffy CJ, Roy JP (1990) Functional specialization for visual motion processing in primate cerebral cortex. Cold Spring Harb Symp Quant Biol 55:717-727.

Xiao DK, Raiguel S, Marcar V, Koenderink J, Orban GA (1995) Spatial heterogeneity of inhibitory surrounds in the middle temporal visual area. Proc Natl Acad Sci USA 92:11303-11306.

Zigler E, Levine J (1981) Premorbid competence in schizophrenia: what is being measured? J Consult Clin Psychol 49:96-105. 